\title{
Nondestructive evaluation of surface coating adhesion using model studies and ultrasonic goniometer experiments
}

\author{
Hansen, Peder Bent; Jensen, Leif Bjørnø
}

Published in:

IEEE Ultrasonics Symposium Proceedings

Link to article, DOI:

10.1109/ULTSYM.1989.67164

Publication date:

1989

Document Version

Publisher's PDF, also known as Version of record

Link back to DTU Orbit

Citation (APA):

Hansen, P. B., \& Jensen, L. B. (1989). Nondestructive evaluation of surface coating adhesion using model studies and ultrasonic goniometer experiments. In IEEE Ultrasonics Symposium Proceedings (Vol. Volume 2, pp. 1125-1128). IEEE. https://doi.org/10.1109/ULTSYM.1989.67164

\section{General rights}

Copyright and moral rights for the publications made accessible in the public portal are retained by the authors and/or other copyright owners and it is a condition of accessing publications that users recognise and abide by the legal requirements associated with these rights.

- Users may download and print one copy of any publication from the public portal for the purpose of private study or research.

- You may not further distribute the material or use it for any profit-making activity or commercial gain

- You may freely distribute the URL identifying the publication in the public portal 


\title{
NONDESTRUCTIVE EVALUATION OF \\ SURFACE COATING ADHESION USING MODEL STUDIES AND ULTRASONIC GONIOMETER EXPERIMENTS
}

\author{
Peder Bent Hansen, Leif Bjørnø \\ Industrial Acoustics Laboratory \\ Technical University of Denmark \\ Building 425, 2800 Lyngby, Denmark
}

\begin{abstract}
A general purpose model for studying the reflection of acoustic plane waves or line focused beams from a stratified medium is presented. Loss of adhesion between one or more layers is taken into account by assuming a partial linear tangential displacement slip between the layers. Measurements of the reflection of focused ultrasonic beams from a aluminium plate covered by a thin self-adhesive aluminium foil, whose adhesion to the aluminium plate locally is decreased, are presented. The measured reflection coefficients are compared to calculated beam reflection coefficients, and the numerical model is used for interpreting the measured results.
\end{abstract}

\section{Introduction.}

Surface coatings are today an important factor in many industrial products because they enhance the quality of the product, e.g. the corrosion- or wear resistance. Therefore, a need exists for a non-destructive method for evaluation of surface coating quality.

Depending on the actual type, a surface coating can consist of one or more layers of the same or different materials (metallic, organic, ceramic). Of the different types of defects (adhesive, cohesive) which lower the quality of the coating, we are mainly interested in detection of adhesive defects, because they cannot be found by visual inspection methods, and because they give rise to a Weak Boundary Layer (WBL), which is often the reason for a later delamination of the coating.

Detection of WBL by means of ultrasonic inspection methods is complicated due to several reasons. First of all WBL does not give rise to local changes in the acoustic impedance like in form of for instance voids. Secondly, WBL's are located between or near layers whose material parameters and thicknesses (normally below $100 \mu \mathrm{m}$ ) depend much on the actual type of coating, which often are partly unknown. The last item means that the wave types which travels in or near a coating in combination with a given substrate can vary strongly from one type of a coating to another. Thus, the way reflection of ultrasonic waves are influenced by the presence of a WBL can be totally different from one type of coating to another.

As a part of the work behind this paper a theoretical general purpose model for studying the reflection of ultrasonic beams / plane waves from a stratified medium is developed. The model is used for estimating material parameters by comparing calculated and measured results. Moreover, an interpretion of the wave type generated in the medium is done and observed resonance phenomena are discussed. The model is also used as a tool for choosing an approach for measuring the presence of a WBL in a particular sample, and for later evaluation of the measured results.

In this paper the the developed numerical model is verified by comparing the calculated and measured effects by the reflection of a focused beam from a aluminium sample covered with a two layer coating, when a WBL is introduced between the coating and the substrate.

\section{Theoretical model.}

The transfer matrix method, also known as the Thomson-Haskell matrix method, is used as a basis for the theoretical studies. The original formulation [1],[2] is generalized, to a form which permits a calculation of the reflection of a line focused acoustic beam from multilayered viscoelastic media. The presence of a WBL between two layers is modelled by assuming a linear tangential displacement slip between the layers. In the following paragraph the main features of the theory used in the developed numerical model is presented. Let us consider $n-1$ viscoelastic layers, bounded below by a viscoelastic solid half-space and above by a liquid half-space as shown in Fig. 1.

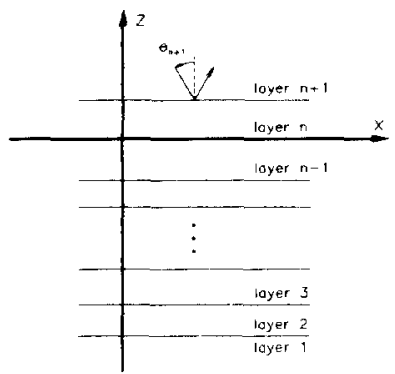

Fig. 1: Geometry of the multilayered system. For layer $l$ is known: $c_{l}=$ longitudinal velocity, $b_{l}=$ transverse velocity, $\rho_{l}=$ density.

A plane harmonic wave is assumed to be incident on the layers from the liquid half-space with an angle of incidence of $\boldsymbol{\theta}_{n+1}$. For any layer $l$, a potential function $\phi_{l}$ for longitudinal waves and a potential function $\Psi_{I}$ for transverse waves may be defined.

$$
\begin{aligned}
& \phi_{l}=\left[A_{l} e^{\left(i \alpha_{l} z\right)}+B_{l} e^{\left(-i \alpha_{l} z\right)}\right] e^{\left|i\left(\sigma_{x} x-\omega\right)\right|} \\
& \Psi_{l}=\left[C_{l} e^{\left(i \beta_{l} z\right)}+D_{l} e^{\left(-i \beta_{l} z\right)}\right] e^{\left|i\left(\sigma_{x} x-\cos \right)\right|}
\end{aligned}
$$

where

$$
\alpha_{l}=\sqrt{\left(k_{l}^{2}-\sigma_{x}^{2}\right)} ; \beta_{l}=\sqrt{\left(\kappa_{l}^{2}-\sigma_{x}^{2}\right)}
$$

and where $\sigma_{x}=k_{l} \sin \theta_{1}$ is the horizontal component of the wave vector and the same for all layers (Snell's law). Attenuation in the layers is introduced by making the wavenumbers $k_{l}$ and $\kappa_{l}$ 
complex via the approach developed in [3]. $A_{l}, B_{l}, C_{l}$ and $D_{l}$ are the amplitude coefficients. The velocity components $v_{x}, v_{z}$ and the stress components $Z_{x}, Z_{z}$ in an arbitrary layer can be expressed in terms of the potential functions

$$
\left[\begin{array}{l}
v_{x}^{(l)} \\
v_{z}^{(l)} \\
Z_{z}^{(l)} \\
Z_{x}^{(l)}
\end{array}\right]=\left\lfloor a_{l}\right\rfloor\left[\begin{array}{l}
A_{l}+B_{l} \\
A_{l}-B_{l} \\
C_{l}-D_{l} \\
C_{l}+D_{l}
\end{array}\right]
$$

The elements of the $4 \times 4$ matrix $[a]$ can be found in [4]. The standard approach in order to determine the reflection coefficient is first to eliminate the amplitude coefficients for all the layers by using the boundary conditions between the layers requiring continuity of normal- and tangential stresses and normal and tangential velocities. This procedure yields a relation between the velocities and stresses at the top and bottom boundaries. By utilizing the boundary conditions between the upper halfspace and the top layer it is then possible to get an equation, from which the reflection coefficient $R\left(\sigma_{x}\right)=A_{n+1}$ can be found [4], [5].

A method to represent a weak boundary layer is incorporated into the model by using the assumptions described by Murty [6], that a weakly bonded interface can be described as a tangential displacement slip with a linear relation between the slip and the shear stress at the interface. These assumptions can for interface $l$ at the bottom of layer $l+1$ be written as

$$
Z_{x}^{(l)}=k_{T}^{(l)}\left(u_{+}^{(l)}-u_{-}^{(l)}\right)=\frac{1}{i \omega} k_{T}^{(l)}\left(v_{+}^{(l)}-v_{-}^{(l)}\right)
$$

where " + " indicates the upper and "-" the lower sides of the interface, and $k_{T}^{(l)}$ is the tangential stiffness of the interface, which according to [6] can be expressed as

$$
k_{T}^{(l)}=i \omega \rho^{(l+1)} b^{(l+1)}\left(\frac{B_{p}^{l}}{1-B_{p}^{l}}\right)
$$

The parameter $B_{p}^{l}$ is a normalized bonding parameter for interface $l$, ranging form 0 to 1 . When $B_{p}^{l}=0$, no bonding exists between the two layers surrounding the interface, and when $B_{p}^{l}=1$ the bonding is perfect. For all other values of $B_{p}^{l}$ a weakened bonding between the layers exits. It should be emphasized that the slip interface at all angles of incidence, except normal, acts as an absorber of acoustic wave energy.

As a way of adapting the numerical calculations to the actual performed experiments, the reflection of a line focused or a non-focused monochromatic acoustic beam from the layered media is incorporated in the model. This is done by representing the reflected beam as a spectral superposition of reflected plane waves. This can be expressed by the following pair of Fourier integral transforms [7] :

$$
\begin{aligned}
\operatorname{Uref}(x, z) & =\frac{1}{2 \pi} \int_{-\infty}^{\infty} R\left(\sigma_{x}\right) V\left(\sigma_{x}\right) e^{i\left(\sigma_{x} x-\sigma_{z} z\right)} d \sigma_{x} \\
V\left(\sigma_{x}\right) & =\frac{1}{2 \pi} \int_{-\infty}^{\infty} U_{i n c}(x, 0) e^{i\left(\sigma_{x} x\right)} d \sigma_{x}
\end{aligned}
$$

where $U_{\text {ref }}(x, z)$ is the field distribution of the reflected beam, $U_{i n c}(x, 0)$ the field distribution of the incidence beam at the interface between the liquid halfspace and the top layer, and $\sigma_{x}$ and $\sigma_{z}$ are the $x$ and $z$ components of the wave vector. In the calculations the Fourier integrals are solved by standard FFT routines. The field distribution $U_{i n c}(x, 0)$ is calculated by the following curve integral

$$
U_{i n c}(x, 0)=\frac{1}{2 \pi} \int_{C} v_{C} \frac{e^{i k r}}{r} d C
$$

where $v_{C}$ is the surface velocity at the source point and $r$ is the distance between the source point and the observation point. The beam reflection coefficient is calculated by integrating the reflected field at the surface of the receiver transducer and dividing it with the emitted field at the surface of the transmitter transducer. Compared to the plane wave reflection coefficient the beam reflection coefficient is lower because it takes into account the attenuation of the beam during propagation in the upper halfspace and scattering of the beam. The beam reflection coefficient is varying less at most angles of incidence than the plane wave reflection coefficient, because it can be interpreted as an average of the plane wave reflection coefficients around the angle of incidence. Around special angles of incidence, like the Rayleigh angle, the beam reflection coefficient shows considerable deviation from the plane wave reflection coefficient, because it takes into consideration the shift of the reflected beam from the position predicted by geometry and the phase shift within the reflected beam, that is created by interference phenomenon (Fig. 3).

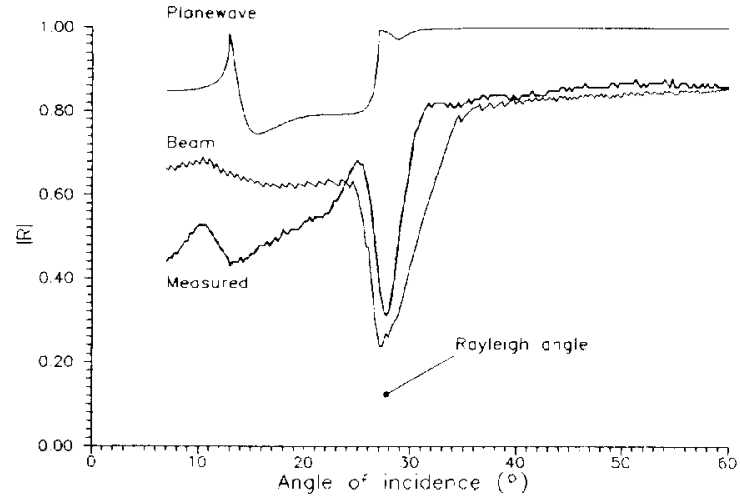

Fig. 2: Calculated plane wave and focused beam reflection coefficients versus angle of incidence for a halfspace of aluminium. compared with the measured intensity of the reflected beam from a thick aluminium plate.

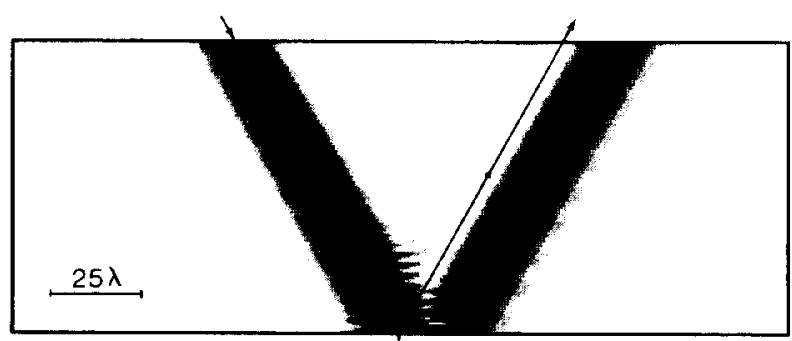

Fig. 3: Calculated field distribution of an incoming focused beam and the reflected beam at the Rayleigh angle of an aluminium halfspace. Notice the shift in the position of the reflected beam and intensity minimum in the reflected beam. 


\section{Experimental Set-up.}

For the experiments a computerized ultrasonic goniometer system is used. The transmitter and receiver transducers are adjusted to equal angles from normal incidence in a pitch-catch configuration. The transmitter transducer is driven by a tone burst signal generated by a function generator. The reflected signal measured by the receiver transducer is acquired using a digital oscilloscope and sent to a PC-computer (Fig. 4). For all measurements presented is this paper, receiver and transmitter transducer used are point focusing transducers with an 1/4" diameter and a 2" focal length. The transmitter transducer is driven by a 15 pulses tone burst signal with a centre frequency of $12.4 \mathrm{MHz}$ and the peak-peak amplitude of the reflected signal is aquired.

As test samples for the measurements presented in this paper $29 \mathrm{~mm}$ thick polished aluminium plates covered with a self-adhesive aluminium foil are used. The self-adhesive foil is considered as a two layer coating consisting of an approximately $55 \mu \mathrm{m}$ thick glue layer covered by an approximately $75 \mu \mathrm{m}$ thick aluminium layer. The material parameters of the glue are unknown and must be estimated based on the measurements. $A$ weak boundary layer WBL is introduced locally by contaminating the surface of the aluminium plate with a very thin layer of ball bearing grease, before the coating is applyed..

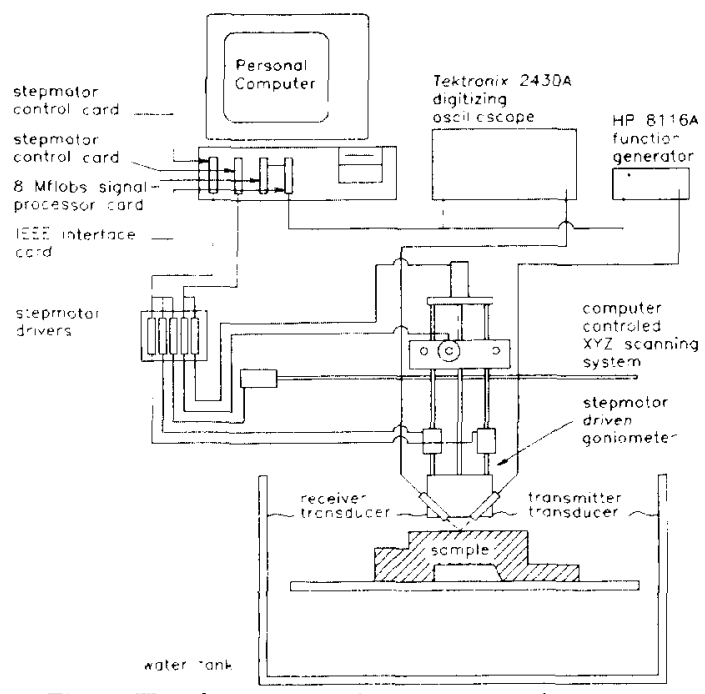

Fig. 4: The ultrasonic goniometer measuring system.

\section{Discussion of numerical and experimental results.}

First a few general things about the calculated results. For all beam calculations the width of the transducers is set to $54.3 \lambda$ and the focal length set to $434 \lambda$, corresponding to a $1 / 4$ ' 'transducer with a 2" focal length at $12.4 \mathrm{MHz}$ used in water. The surface velocity of the transmitter transducer is assumed to have a Gaussian distribution. The transducers are placed in the liquid halfspace so their geometrical focuses are at the same point at the surface of the top layer.

In figure 2, the measured results for the reflection of acoustic waves from a aluminium halfspace are shown. The measured curve, like all the other measured curves shown here, is multiplied by a factor, so it equals the calculated results at the angle of incidence equal to $60^{\circ}$. The small fluctuations on the beam reflection coefficient curve is due to the discrete nature of the calculations. We see that the beam calculations are capable of describing the big drop in reflection coefficient around the
Rayleigh angle which is measured. We also observe an increasing deviation between the measured and calculated reflection coefficients, when the angle of incidence is deceasing. This is an occurrence, which is observed in all the measurements performed and we believe it is a problem, that has something to do with the adjustment of the goniometer system and not the numerical model. Lack of time has prevented us from investigating the problem more thoroughly.

In figure 5 the measured and calculated curve for the reflection coefficient from the aluminium plate covered with the self-adhesive aluminium foil is shown. The bonding is assumed to be perfect. The parameters of the glue are adjusted to make the calculated curve fit the measured curve. We notice a very good agreement is obtained for angles of incidence above $25^{\circ}$.

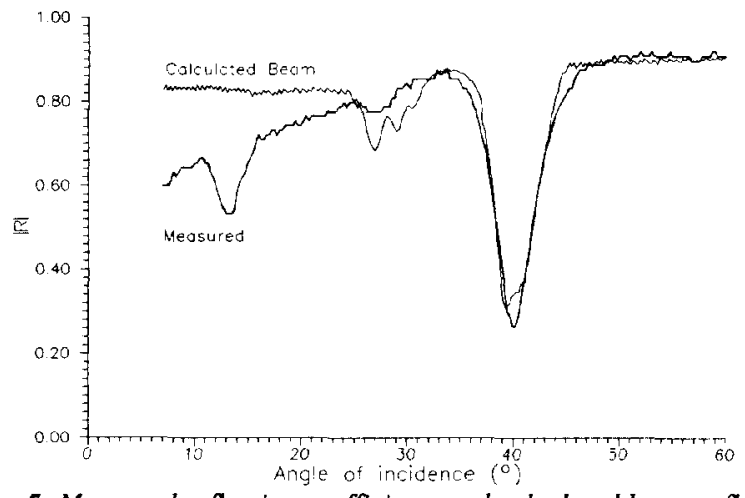

Fig. 5: Measured reflection coefficients and calculated beam reflection coefficient versus angle of incidence for test sample with good bonding.

Special attention must be drawn to the drop in reflection coefficient around $40^{\circ}$, because it is due to the surface coating. The numerical model is used to determine the reason for the drop in the reflection coefficient. During the estimation process the size and location of the drop showed great sensitivity to both the longitudinal and the shear velocity and thickness of the glue. This dependence of all the parameters is explained by looking at a calculated field distribution of the reflected beam from the medium at $40^{\circ}$ angle of incidence (fig. 6 ).

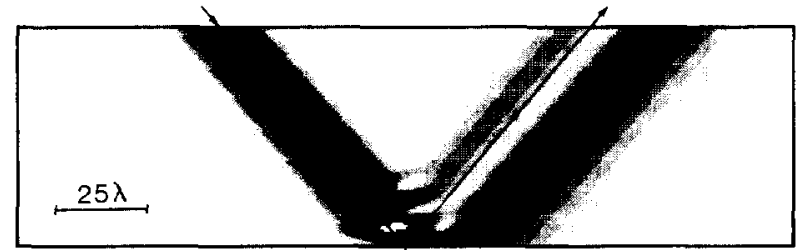

Fig. 6: Calculated field distribution of an incoming focused beam and the reflected beam at $40^{\circ}$ angle of incidence. Beam reflected from an aluminium halfspace covered with a $67 \mu \mathrm{m}$ thick glue layer and a $75 \mu \mathrm{m}$ thick aluminium layer.

From fig. 6 we can see, that the drop in the reflection coefficient is caused by a displacement of the reflected beam and a phase shift in the reflected beam. The explanation for this is, the main part of the beam energi, which is transmitted into the glue layer at this angle of incidence is trapt in the glue layer, because the glue layer is bounded by layers with higher sound velocities. When the wave trapt in the glue layer propagates, is it attenuated due to the attenuation and scattering of energi into the aluminium halfspace and back into the water. Interference between the part of the beam reflected from the interface between the water and the aluminium foil and the wave scattered back into water causes 
the phase shift in the refelcted beam and the minimum zone in the reflected beam.

The measured reflection coefficients for an area of the test sample with good and an area of the test sample with weakened bonding is shown in fig. 7. A significant deviation between the two curves are seen around $40^{\circ}$ angle of incidence and what is also interesting to see, that no deviation is observed at low angles of incidence. We see that the minimum of the refelction coefficient is less sharp, and the explanation could be, that the WBL changes the boundary conditions in a way, that makes beam energi be trapt in the glue layer at more angles of incidence, but also makes more energi scatter back into the water.

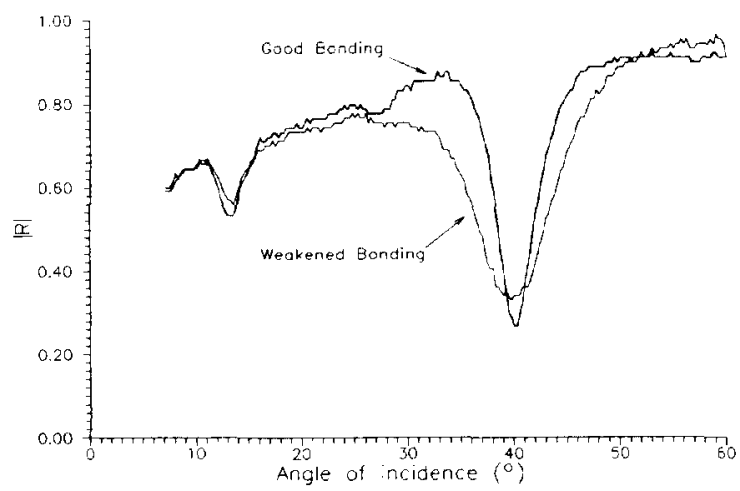

Fig. 7: Measured reflection coefficients versus angle of incidence for an area of the test sample with good bonding and an area with weakened bonding.

Curves for the reflection coefficient calculated for different values of the bonding parameter $B_{p}$ belonging to the interface between the aluminium halfspace and the glue are shown in Fig. 8.

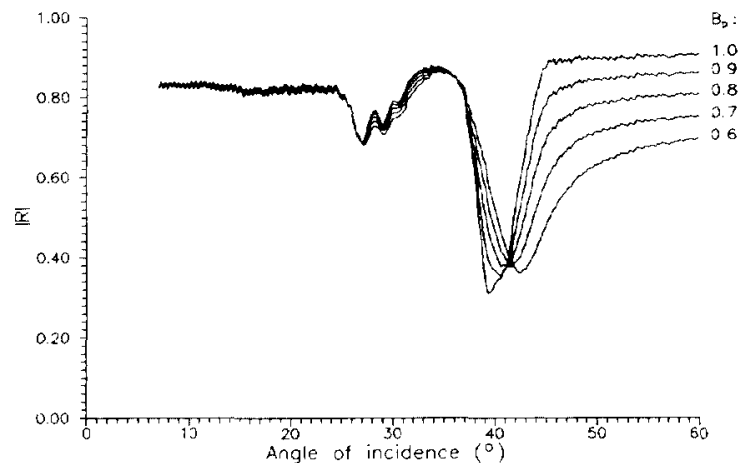

Fig. 8: Calculated beam reflection coefficients versus angle of incidence for different values of the bonding parameter $B_{p}$ belonging to the interface between the aluminium halfspace and the glue.

We see an good agreement between the measured and the calculated results considering the insensitivities of the reflection coefficient to a WBL at low angles of incidence. We also observe the model predicts a reduction in the depth of drop in reflection coefficient around $40^{\circ}$ angle of incidence, at least at moderate decreases of the bonding parameter. Contrary to measurements the calculated position of the minimum of drop moves to greater angles of incidence, and the reflection coefficient is in general lowered at great angles of incidence, when a WBL is introduced.

\section{Conclusion.}

By use of a ultrasonic goniometer system the reflection of focused ultrasonic beams from a aluminium plate covered with a two layer coating is measured. The measurement have shown, that the reflection at special angles show significant sensitivity to the presence of a weak boundary layer between the coating and the substrate. A numerical model developed for calculating beam reflection coefficients has proved to be a powerful tool for estimating missing material parameters and assisting interpretation of the measured results. The approach describing a weak boundary layer as a linear tangential displacement slip between the layers has only been a limited success. Before the numerical model can be used to evaluate the decrease in bonding strength due to WBL must a new approach should therefore be developed.

\section{References.}

'Thomson W.T.: Transmission of Elastic Waves Through a Stratified Solid Medium, Jour. Appl. Phys., 21, 89-93 (1950)

${ }^{2}$ Haskell N.A.: The Dispersion of Surface Waves on Multilayered Media, Bull. Seismol. Soc. Am., 43, 17-43 (1953)

${ }^{3}$ Madigosky W., Fiorito R.: Model Resonance Analysis of Acoustic Transmission and Reflection Losses in Viscoelastic Plates, J. Acoust. Soc. Am., 65, 1105-1115 (1979)

${ }^{4}$ Folds D., Loggins C.: Transmission and Reflection of Ultrasonic Waves in Layered Media, J. Acoust. Soc. Am., 62, 1102-1109 (1977)

s Brekhovskikh L.M.: Waves in Layered Media, Second Edition, Academic Press (1980)

${ }^{6}$ Murty G.S: A Theoretical Model for the Attenuation and Dispersion of Stoneley Waves at the Loosely Bonded Interface of Elastic Half Spaces, Physics of the Earth and Planetary Interiors, 11, 65 (1975)

' Ngoc T.D.K., Mayer W.G.: A General Description of Ultrasonic Nonspecular Reflection and Transmission Effect for Layered Media, IEEE Trans. Sonics Ultrason., SU-27,5,229-236 (1980) 